\section{Mensuração do impacto dos problemas bucais sobre a qualidade de vida de crianças: aspectos conceituais e metodológicos}

\author{
Measuring the impact of oral health problems on \\ children's quality of life: conceptual and \\ methodological issues
}

\author{
${ }^{1}$ Faculdade de Odontologia, \\ Universidade do Estado do \\ Rio de Janeiro, Rio de Janeiro, \\ Brasil. \\ 2 Faculdade de Odontologia, \\ Universidade Federal do Rio \\ de Janeiro, Rio de Janeiro, \\ Brasil. \\ Correspondência \\ F. C. Tesch \\ Departamento de \\ Odontologia Preventiva e \\ Comunitária, Faculdade de \\ Odontologia, Universidade \\ do Estado do Rio de Janeiro. \\ Rua Engenheiro Durval de \\ Souza 171, Petrópolis, RJ \\ 25710-320, Brasil. \\ fatesch@hotmail.com
}

\begin{abstract}
Children are subject to oral health problems that can impact their own quality of life and that of their families. The need for measurements to assess the impact of oral health on children's quality of life has been emphasized, and questionnaires have been developed or adapted to this specific group. The aim of this study was to describe such instruments (identified in the literature) and discuss their inherent difficulties. Among such instruments, we highlight the Child Oral Health Quality of Life Questionnaire, for children aged 6-7, 8-10, and 11-14, the Child-Oral Impacts on Daily Performances, for children aged 11-12, and the Early Childhood Oral Health Impact Scale for children aged 2-5. Although progress was observed in this area, more research is needed to develop measurements that apply to children in their dynamic health/disease process, encompassing their psychosocial, family, and social context. The use of such instruments should be encouraged in clinical practice and research.
\end{abstract}

Oral Health; Child Health; Quality of Life
Flávia Cariús Tesch 1

Branca Heloísa de Oliveira 1

Anna Leão 2

\section{Introdução}

A saúde bucal é parte da saúde geral e é essencial para a qualidade de vida. Todos os indivíduos devem dispor de uma condição de saúde bucal que lhes permita falar, mastigar, reconhecer o sabor dos alimentos, sorrir, viver livre de dor e desconforto, e se relacionar com outras pessoas sem constrangimento ${ }^{1}$. As pessoas percebem a importância da saúde bucal para a qualidade de vida sob uma variedade de formas nos domínios físico, social e psicológico, sendo que a capacidade de se alimentar e a ocorrência de dor e desconforto costumam ser considerados os aspectos positivo e negativo mais relevantes para a qualidade de vida, respectivamente 2 .

Apesar das grandes conquistas associadas à saúde bucal nas últimas décadas, muitas pessoas em todo o mundo, especialmente as mais pobres, ainda são afetadas por problemas bucais como a cárie e a doença periodontal 1 . Na infância, a cárie dentária é considerada a doença mais comum dentre aquelas que não regridem espontaneamente e nem são passíveis de cura por intervenções farmacológicas de curto prazo ${ }^{3}$. A cárie afeta de $60 \%$ a $90 \%$ das crianças em idade escolar nos países industrializados e também é uma doença muito prevalente entre as crianças asiáticas e latino-americanas 1 . No Brasil, a cárie dentária acomete $27 \%$ das crianças entre 18 e 36 meses de idade, sendo que este percentual chega a $59,4 \%$ aos cinco anos de idade. Entre as crianças de 12 
anos, $70 \%$ possuem pelo menos um dente permanente cariado e, entre os adolescentes de 15 a 19 anos, $90 \%$ apresentam a doença ${ }^{4}$. Os efeitos negativos da cárie dentária sobre a vida das crianças incluem: dificuldade de mastigar, diminuição do apetite, perda de peso, dificuldade para dormir, alteração no comportamento (irritabilidade e baixa auto-estima) e diminuição do rendimento escolar 5,6,7,8,9,10. Foi relatado ainda que, quando uma criança apresenta um quadro de cárie rampante na primeira infância, o bemestar da família pode ser comprometido: os pais sentem-se culpados, e, por vezes, precisam faltar ao trabalho e ter despesas com o tratamento odontológico da criança 11.

Outros problemas bucais, como os transtornos associados à erupção dentária 12, os traumatismos dentários 13 , as patologias dos tecidos moles da boca 12 , as oclusopatias 14 , as fissuras labiopalatais 15 e a fluorose dentária 16 afetam crianças com freqüência variável. Entretanto, existe pouca informação a respeito das suas possíveis conseqüências funcionais, emocionais e sociais $17,18,19,20$.

Apesar de, em 1985, Nikias 21 ter sugerido que a avaliação do efeito das doenças bucais sobre a qualidade de vida pode ser muito útil para auxiliar planejadores de serviços de saúde a estabelecer programas e prioridades institucionais, pesquisadores a estudar os determinantes do processo saúde/doença e a identificar medidas terapêuticas e profiláticas realmente efetivas, e clínicos a tomar decisões de tratamento e a monitorar a evolução clínica de seus pacientes, o primeiro trabalho descrevendo o processo de construção e validação de um questionário para mensurar a qualidade de vida relacionada à saúde bucal de crianças foi publicado somente em 2002 22,23.

Além da utilização de instrumentos desenhados especificamente para avaliar a qualidade de vida relacionada à saúde bucal de crianças ter sido iniciada recentemente, praticamente não existe informação científica publicada no nosso idioma sobre o assunto, o que limita a ampliação do emprego clínico e em pesquisa, no Brasil, desse tipo de instrumento.

Este trabalho tem por objetivo descrever os indicadores de qualidade de vida associada à saúde bucal de crianças publicados na literatura científica, e discutir aspectos conceituais e metodológicos associados à utilização dos mesmos. Os indicadores de qualidade de vida incluídos foram selecionados a partir de buscas nas bases de dados LILACS (Literatura Latino-americana em Ciências de Saúde), BBO (Bibliografia Brasileira de Odontologia) e MEDLINE (Medical Literature Analysis and Retrieval System Online), compreendendo o período de 1980 até 2006, e na base eletrônica de resumos da International Association for Dental Research, no período de 2001 a 2006, empregando-se os termos "quality of life", "children", "oral health", "health assessment" e "questionnaires", combinados ou não. Além disso, foram consultadas as listas de referências bibliográficas dos artigos científicos localizados a partir da estratégia de busca anteriormente mencionada.

\section{Indicadores de qualidade de vida associada à saúde bucal}

A qualidade de vida pode ser definida como " $a$ percepção do indivíduo de sua posição na vida, no contexto da cultura e do sistema de valores nos quais ele vive, e em relação aos seus objetivos, expectativas, padrões e preocupações" 24 (p. 1). O conceito de qualidade de vida, além de compreender uma representação subjetiva da sensação de bem-estar, é multidimensional e inclui tanto dimensões positivas quanto negativas 25,26,27. Na década de 90 consolidou-se a idéia que, em função dessas características, os instrumentos de mensuração da qualidade de vida devem considerar a perspectiva das pessoas e das populações e não se restringir à perspectiva de profissionais de saúde e pesquisadores 26 .

Geralmente, os indicadores de qualidade de vida associada à saúde são construídos sob a forma de questionários compostos de itens (perguntas) que procuram medir, por meio de respostas organizadas sob a forma de escalas numéricas, o quanto aspectos da vida das pessoas, nos domínios físico, psicológico, material e social, entre outros, são afetados pelas condições de saúde 28,29. Logo, um aspecto importante desse tipo de ferramenta é a sua capacidade de representar sob a forma numérica as diferenças entre pessoas e comunidades no que diz respeito à qualidade de vida associada à saúde 29 . Porém, não se pode perder de vista que qualidade de vida é um construto que, como tal, não pode ser completamente operacionalizado e diretamente medido. Assim, é importante ressaltar que as medidas numéricas obtidas com a aplicação dos indicadores de qualidade de vida associada à saúde devem ser sempre consideradas índices imperfeitos de um construto subjacente 28 . Os indicadores de qualidade de vida associada à saúde genéricos avaliam a qualidade de vida relacionada à saúde geral e incluem uma variedade de alterações funcionais e incapacidades que podem afligir as pessoas 30 . Dentre os instrumentos genéricos mais freqüentemente utilizados podemos citar o WHOQOL-100 (Instrumento de Avaliação da Qualidade de Vida da 
Organização Mundial da Saúde) 31, o SF-36 (Medical Outcomes Study Questionnaire) 32,33 e o SIP (Sickness Impact Profile) 34 . Já os instrumentos específicos, focalizam uma determinada população, função, doença, condição ou problema, ou seja, um determinado grupo de pessoas com necessidades específicas 30,35. Dentre os questionários específicos desenvolvidos para crianças podemos citar: o AUQEI (Autoquestionnaire Qualité de Vie Enfant Imagé) 27,36 e o CHQ (Child Health Questionnaire) 35,37,38.

Os questionários que se destinam a avaliar o impacto dos problemas bucais sobre a qualidade de vida são instrumentos específicos denominados genericamente de indicadores sócio-dentais 39,40. Suas aplicações são amplas e incluem ações políticas, de pesquisa, de saúde pública e clínicas $41,42,43$.

Nas últimas duas décadas, vários indicadores sócio-dentais foram desenvolvidos, como o GOHAI (Geriatric Oral Health Assessment Index) 44, o DIDL (Dental Impacts on Daily Living) 45, o OHIP (The Oral Health Impact Profile) 46 e a sua versão abreviada - o OHIP-14 47 e o OIDP (Oral Impacts on Daily Performances) 48. Esses questionários foram construídos para serem aplicados na população adulta, porém, recentemente, tanto o OHIP quanto o OIDP foram utilizados em estudos envolvendo adolescentes 17,49,50,51.

Apesar de indicadores sócio-dentais elaborados para adultos terem sido aplicados com sucesso em adolescentes, a percepção de adultos e crianças a respeito do impacto dos problemas de saúde sobre a qualidade de vida é diferente, já que as crianças e os adolescentes possuem uma visão peculiar de si mesmos e do mundo devido à fase de desenvolvimento físico e emocional em que se encontram 27,30,52. Portanto, o desenvolvimento de instrumentos específicos para crianças viabiliza a mensuração mais acurada do impacto dos problemas bucais sobre a sua qualidade de vida. Levando isso em consideração, Jokovic et al. 22 construíram o COHQOL (Child Oral Health Quality of Life Instrument), Broder et al. 53 desenvolveram o COHIP (Child Oral Health Impact Profile), Gherunpong et al. ${ }^{54}$ adaptaram o OIDP para uso em crianças criando o CHILD-OIDP, e Talekar et al. 55 desenvolveram o ECOHIS (The Early Childhood Oral Health Impact Scale) (Tabela 1).

Tanto o COHQOL quanto o ECOHIS baseiamse no modelo conceitual proposto por Locker 56 (Figura 1). Esse autor desenvolveu um esquema que conecta de forma linear os conceitos de doença, deficiência, limitação funcional, incapacidade e desvantagem social, permitindo que a análise do impacto dos problemas bucais sobre a vida das pessoas seja realizada de forma progressiva, do nível biológico para o comportamental e deste para o social.

O COHQOL é um questionário que objetiva mensurar a qualidade de vida relacionada à saúde bucal de crianças com idades entre 6 e 14 anos. Esse instrumento inclui um questionário sobre o impacto nos pais e na família dos problemas bucais das crianças (Family Impact Scale - FIS), um questionário para mensurar a percepção da criança sobre a sua própria saúde bucal (Child Perception Questionnaire-CPQ) que varia segundo a faixa etária da criança, e um questionário para mensurar a percepção dos responsáveis sobre a saúde bucal dos filhos (Parental Perceptions Questionnaire - PPQ) 22,57,58. Recentemente, os autores testaram a possibilidade de reduzir o número de itens do CPQ para crianças de 11 a 14 anos e concluíram que, para facilitar o uso na clínica e em inquéritos epidemiológicos, é possível empregar, nesta faixa etária, versões do questionário com apenas 8 ou 16 itens 59 .

O ECOHIS 55 inclui itens originados do COHQOL que foram testados e considerados relevantes para avaliar a qualidade de vida relacionada à saúde bucal de crianças com idades entre 2 e 5 anos. $O$ instrumento possui uma subescala para a criança e outra para a família e deve ser preenchido pelos responsáveis das crianças. Ele acessa tanto o impacto da saúde bucal na vida diária da criança, como o impacto relativo aos tratamentos dentários que ela possa ter realizado. O ECOHIS foi traduzido para o idioma espanhol 60 e foi adaptado para o idioma português, no Brasil 61, e para o idioma francês, no Canadá 62 , demonstrando ser válido e confiável em culturas distintas.

O CHILD-OIDP 54 foi desenvolvido com base no OIDP, sendo que as modificações realizadas visaram a adequar o questionário para crianças de 11 a 12 anos de idade. O CHILD-OIDP tem por base um modelo conceitual diferente do utilizado na construção do COQOL e do ECOHIS, e assim como o OIDP, ele foi construído somente com impactos sobre a capacidade de executar as atividades da vida diária relativas aos desempenhos físico, psicológico e social 54,63. O CHILDOIDP possui uma lista com todos os problemas bucais que as crianças são capazes de perceber e utiliza figuras para ilustrar as perguntas. As crianças são solicitadas a identificar quais desses problemas elas apresentaram nos três meses anteriores, a relatar se os problemas experimentados causaram alguma dificuldade para realizar atividades da vida diária e indicar a freqüência e a gravidade com que a dificuldade ocorreu. Os autores observaram que o CHILD-OIDP, aplicado a crianças tailandesas com idades de 11 a 12 anos, 
Tabela 1

Características dos instrumentos para a avaliação do impacto dos problemas bucais sobre a qualidade de vida de crianças.

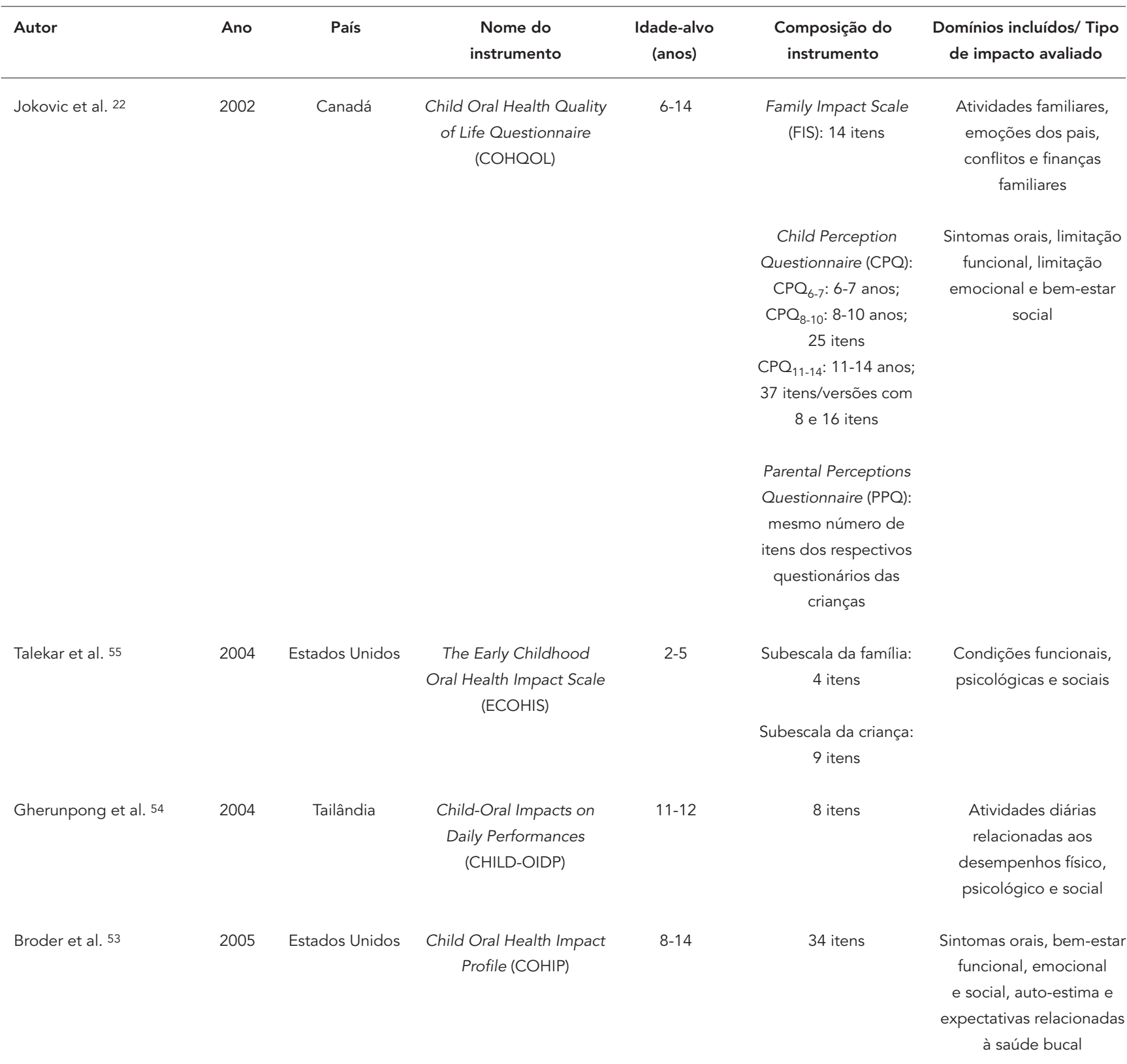

era válido e confiável. Tubert-Jeannin et al. 64 produziram a versão francesa do CHILD-OIDP que manteve as propriedades psicométricas do instrumento original.

O COHIP 53 foi elaborado para ser respondido pelos pais e pelas crianças e inclui questões que avaliam tanto aspectos positivos quanto negativos da qualidade de vida relacionada à saúde bucal, o que lhe confere um caráter inovador em relação aos instrumentos anteriormente citados.
Infelizmente, há escassez de informação sobre o COHIP na literatura odontológica.

No que diz respeito às propriedades psicométricas dos indicadores sócio-dentais desenvolvidos para crianças, os autores têm se preocupado em relatar tanto medidas de confiabilidade quanto de validade (Tabela 2). A confiabilidade desses instrumentos tem sido acessada em termos de consistência interna e estabilidade. Com relação à validade, tem-se procurado examinar 


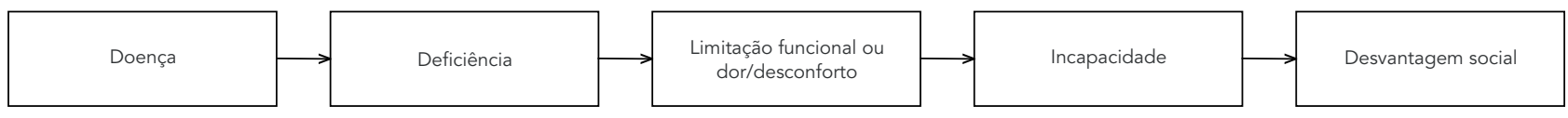

Tabela 2

Confiabilidade e validade dos instrumentos de avaliação da qualidade de vida associada à saúde bucal de crianças

\begin{tabular}{|c|c|c|c|}
\hline Instrumentos & $\begin{array}{l}\text { Consistência } \\
\text { interna (alfa } \\
\text { Cronbach) }\end{array}$ & $\begin{array}{l}\text { Estabilidade } \\
\text { (teste- } \\
\text { reteste) }\end{array}$ & Validade \\
\hline \multicolumn{4}{|l|}{ Child Oral Health Quality of Life Questionnaire } \\
\hline \multicolumn{4}{|l|}{ (COHQOL) } \\
\hline \multicolumn{4}{|l|}{ Child Perception Questionnaire (CPQ) } \\
\hline $\mathrm{CPQ}_{11-14}$ & 0,91 & 0,90 * & Correlação com a percepção de saúde geral \\
\hline $\mathrm{CPQ}_{8-10}$ & 0,89 & 0,75 * & e o bem-estar global e associação com medidas \\
\hline $\mathrm{CPQ}_{6-7}$ & - & - & normativas de saúde bucal \\
\hline Parental Perceptions Questionnaire (PPQ) & 0,94 & 0,85 * & \\
\hline Family Impact Scale (FIS) & 0,83 & 0,80 * & \\
\hline \multicolumn{4}{|l|}{ Child-Oral Impacts on Daily Performances } \\
\hline \multicolumn{4}{|l|}{ (CHILD-OIDP) } \\
\hline CHILD-OIDP original & 0,82 & $0,93 * \star$ & Associação com a percepção de problemas bucais e com a \\
\hline CHILD-OIDP francês & 0,57 & $0,75 * \star$ & necessidade percebida de tratamento odontológico \\
\hline \multicolumn{4}{|l|}{$\begin{array}{l}\text { Early Childhood Oral Health Impact Scale } \\
\text { (ECOHIS) }\end{array}$} \\
\hline ECOHIS original & 0,87 & 0,84 * & Correlação com as avaliações globais de saúde geral e \\
\hline S-ECOHIS espanhol & 0,81 & - & bucal das crianças feitas pelos pais \\
\hline B-ECOHIS português & 0,80 & 0,98 * & \\
\hline F-ECOHIS francês & 0,76 & - & \\
\hline Child Oral Health Impact Profile (COHIP) & 0,92 & - & $\begin{array}{l}\text { Diferenças estatisticamente significantes entre os escores } \\
\text { dos grupos de pacientes (odontopediátrico, ortodôntico e } \\
\text { com anomalias craniofaciais) com problemas bucais distintos }\end{array}$ \\
\hline
\end{tabular}

* Coeficiente de correlação intraclasse;

** Coeficiente kappa.

em que extensão os conceitos avaliados pelos instrumentos se relacionam com outras variáveis que possam ser medidas de forma subjetiva (por exemplo, avaliação do quanto o bem-estar geral da criança é comprometido pelos problemas bucais em uma escala do tipo Likert) ou objetiva (por exemplo, correlação entre a escala e um índi- ce de cárie ou com a presença de oclusopatia). O $\mathrm{CPQ}_{8-10} 58$ e o $\mathrm{CPQ}_{11-14} 22$ apresentaram boa correlação com a percepção de saúde geral e bemestar global das crianças estudadas. Além disso, as pontuações obtidas com a aplicação desses questionários em crianças com problemas bucais como cárie dentária e oclusopatias também 
foram significativamente maiores do que as pontuações obtidas nas crianças sem problemas bucais. Resultados semelhantes foram obtidos com o ECOHIS 55 e suas versões traduzida para o espanhol 60 e adaptada para o português 61 , e com o CHILD-OIDP 54 e a sua versão francesa 64 .

\section{Desafios para a utilização de questionários para mensurar a qualidade de vida relacionada à saúde bucal em crianças}

Ainda existem muitas questões a serem debatidas e respondidas, como a necessidade de questionários específicos para diferentes idades, a participação dos responsáveis como respondentes secundários e a avaliação do impacto da saúde bucal da criança na família. Esses pontos são descritos a seguir apresentando-se os avanços, as limitações e considerações relativas a cada um deles.

Necessidade de questionários idade-específicos

A percepção de saúde e de doença varia conforme a capacidade cognitiva da criança, a qual se modifica de acordo com a idade, em função dos diferentes estágios de desenvolvimento emocional, social e de linguagem. A percepção da criança também pode variar segundo a sua cultura, a sua condição sócio-econômica e a sua condição de saúde 25,30,65. Para responder a uma pergunta sobre sua qualidade de vida, a criança precisa, primeiramente, entender o que está sendo perguntado e, depois, saber formular uma resposta. A criança também pode ter dificuldade para responder sobre o que viveu e sentiu durante um período de tempo específico, como a semana ou o mês anterior 28. Devido às diferenças de habilidade cognitiva de acordo com a idade, um instrumento elaborado para ser preenchido por uma criança de 11 anos não pode ser utilizado por uma criança de seis anos. Assim, os questionários voltados para a população pediátrica devem ser específicos para cada idade 22,25,66. Entretanto, como os conhecimentos científicos sobre o que as crianças sabem e conseguem relatar em relação à sua saúde ainda são limitados, há necessidade de mais pesquisas que avaliem como a idade, o nível cognitivo, a habilidade de leitura e interpretação, o estado sócio-econômico e o estado de saúde afetam os relatos das crianças 67.

$\mathrm{O}$ fato das crianças viverem um processo contínuo de mudanças também repercute sobre as suas características bucais e sobre a sua aparência facial, dificultando a comparação entre crianças de diferentes idades e a avaliação, nas mesmas crianças, de alterações na qualidade de vida ao longo do tempo 25 .

\section{Utilização de respondentes secundários}

Os questionários desenvolvidos para mensurar a qualidade de vida de crianças devem ser direcionados para diferentes faixas etárias, como de 6 a 7 anos, 8 a 10, 11 a 12, e serem respondidos pelas próprias crianças, pois elas têm o direito de expressar suas opiniões e de tê-las respeitadas 30 . Contudo, alguns grupos de crianças, como as muito jovens, as incapacitadas e as gravemente enfermas, apresentam dificuldade para fornecer informação acurada sobre a sua qualidade de vida 28 . Por esse motivo, os questionários orientados para pré-escolares, por exemplo, costumam ser respondidos pelos responsáveis 55 . Contudo, não se pode garantir que os pais (respondentes secundários) consigam representar bem a realidade das crianças e nem que forneçam respostas verdadeiras, livres de pressão relacionada à aceitabilidade social 25. Para permitir que mesmo crianças jovens possam responder sobre a sua própria qualidade de vida, alguns instrumentos utilizam recursos auxiliares às respostas, como figuras com as quais a criança possa se identificar 27,54 ou estórias desenvolvidas em programas computacionais 68 . Os autores que consideram que crianças em idade pré-escolar não possuem capacidade cognitiva suficiente para avaliar a sua qualidade de vida preferem utilizar como respondentes adultos próximos, de preferência os pais. Dependendo do tipo de informação solicitada no questionário, os relatos dos pais tenderão a ser mais ou menos próximos das experiências vividas pelos filhos, pois foi demonstrado que os pais são capazes de avaliar melhor os domínios relacionados às funções e sintomas físicos do que os domínios relacionados às funções emocionais e sociais 28 . Em geral, os pais tendem a considerar que uma doença tem mais impactos negativos sobre a vida da criança do que a própria criança ${ }^{69}$. No campo específico dos indicadores sócio-dentais, foi realizado um estudo visando a avaliar a concordância entre o PPQ e o $\mathrm{CPQ}_{11-14}$ em um grupo formado por 42 pares de mães/crianças, confirmando-se a tendência de maior concordância entre mães e filhos no que diz respeito a aspectos mais facilmente observáveis, como sintomas orais e limitações funcionais, do que a aspectos nos domínios emocional e social. Entretanto, nesse mesmo estudo, contrariando o que costuma ser relatado na literatura, observou-se que as crianças consideravam a sua qualidade de vida mais comprometida por problemas bucais do que suas mães. 
Um outro aspecto importante a ser considerado, é que pai e criança respondem ao questionário sob perspectivas diferentes e que ambas as informações podem ser relevantes ${ }^{70}$. Portanto, segundo alguns autores, dependendo da faixa etária, seria mais apropriado utilizar os pais como um meio de obter informações adicionais sobre a qualidade de vida da criança $57,66,69,71,72,73$. A forma mais adequada de integrar os relatos dos responsáveis aos das crianças permanece sendo um grande desafio para os pesquisadores da área 67.

Impacto dos problemas bucais sobre a qualidade de vida da família

Crianças não são seres independentes e a família compreende a rede de indivíduos que dá suporte e afeta de forma mais acentuada a qualidade de vida da criança. No sentido inverso, a própria família é afetada pelos problemas de saúde da criança 30 . Familiares de crianças portadoras de doenças crônicas muitas vezes têm suas atividades diárias limitadas e experimentam medos e ansiedades devido aos problemas de saúde da criança. Além disso, os problemas de saúde podem produzir impactos financeiros que tendem a aumentar o estresse familiar 74,75. Portanto, instrumentos desenvolvidos para mensurar a qualidade de vida relacionada à saúde bucal de crianças também devem abordar o impacto desses problemas sobre a qualidade de vida da família ${ }^{23}$. No campo da avaliação da qualidade de vida associada à saúde bucal, por exemplo, o COHQOL 23 apresenta um questionário específico para a família e o ECOHIS 55 possui uma subescala com itens relacionados à família. Esse tipo de recurso permite acessar informações importantes sobre as conseqüências psicológicas, materiais e sociais de problemas de saúde das crianças sobre a família e podem ser muito úteis para o planejamento de serviços de saúde 74 .

\section{Considerações finais}

Pesquisas para avaliar o impacto da saúde bucal na vida das crianças e de seus familiares foram estimuladas pela mudança do conceito de saúde e o conseqüente desenvolvimento de modelos teóricos que associam variáveis biológicas e psicossociais. Nos últimos cinco anos, instrumentos de medida que permitem que a condição de saúde bucal e as necessidades de tratamento odontológico de crianças sejam investigadas sob essa perspectiva têm sido desenvolvidos e testados. Adaptações transculturais de instrumentos já desenvolvidos têm sido realizadas para viabilizar a coleta e comparação dessas informações em diferentes culturas 60,61,64. Estudos empregando esse tipo de instrumento têm registrado o impacto dos diferentes estados de saúde bucal nas crianças, nos pais e na família como um todo 17,18,76. Recentemente, procurou-se também incorporar os possíveis impactos produzidos pelo próprio tratamento odontológico sobre a vida de crianças muito jovens e sobre suas famílias a este tipo de avaliação 55,60,61. Apesar dos progressos no desenvolvimento de medidas de impacto de saúde bucal na qualidade de vida de crianças, ainda há muito para ser estudado. Ainda se precisa saber, por exemplo, quais fatores têm influência realmente relevante sobre a qualidade de vida associada à saúde bucal das crianças sob o ponto de vista das mesmas, como o desenvolvimento físico e psíquico e as experiências relacionadas a outras doenças influenciam a percepção da criança sobre a própria saúde bucal, de que forma a acomodação ao próprio estado de saúde e a tratamentos odontológicos aos quais a criança esteja sendo ou tenha sido submetida agem sobre a percepção do seu bem-estar e até que ponto as informações fornecidas pelos pais ou pessoas próximas são válidas e confiáveis. Finalmente, cabe ressaltar que, neste momento em que a odontologia baseada em evidências vem crescendo em importância, é fundamental que, tanto na clínica quanto na pesquisa, seja substancialmente ampliada a utilização dos instrumentos de avaliação da qualidade de vida associada à saúde bucal, a fim de que desfechos realmente importantes para a vida das crianças e de suas famílias passem a ser considerados. 


\section{Resumo}

As crianças estão sujeitas a problemas de saúde bucal que podem causar impacto na sua vida diária e na vida de suas famílias. A necessidade de instrumentos para acessar o impacto da saúde bucal na qualidade de vida das crianças tem sido ressaltada, e questionários foram desenvolvidos ou adaptados para este grupo específico. O objetivo deste trabalho é descrever os instrumentos de mensuração do impacto da saúde bucal na qualidade de vida de crianças e as dificuldades para esta mensuração. Entre esses instrumentos destacamos o Child Oral Health Quality of Life Questionnaire, para crianças de 6-7, 8-10 e 11-14 anos; $o$ Child-Oral Impacts on Daily Performances, para a idade de 1112 anos e o Early Childhood Oral Health Impact Scale para a faixa etária de 2-5 anos. Apesar dos progressos nessa área, mais estudos são necessários para a elaboração de medidas que abordem a criança dentro do processo dinâmico de saúde e doença no seu contexto psicológico, familiar e social, e a utilização, tanto na prática clínica quanto na pesquisa, dos instrumentos existentes deve ser estimulada.

Saúde Bucal; Saúde da Criança; Qualidade de Vida

\section{Colaboradores}

F. C. Tesch realizou a busca bibliográfica e participou da organização, redação e edição final do artigo. B. H. Oliveira e A. Leão contribuíram para a escolha do tema, organização, redação, correção e edição final do artigo.

\section{Agradecimentos}

Flávia Cariús Tesch foi parcialmente apoiada pelo Conselho Nacional de Desenvolvimento Científico e Tecnológico (CNPq).

\section{Referências}

1. Petersen PE. The World Oral Health Report 2003: continuous improvement of oral health in the 21st century - the approach of the WHO Global Oral Health Programme. Community Dent Oral Epidemiol 2003; 31 Suppl 1:3-23.

2. McGrath C, Bedi R. A national study of the importance of oral health to life quality to inform scales of oral health related quality of life. Qual Life Res 2004; 13:813-8.

3. Vargas CM, Crall JJ, Schneider DA. Sociodemographic distribution of pediatric dental caries: NHANES III, 1988-1994. J Am Dent Assoc 1998; 129:1229-38.

4. Coordenação Nacional de Saúde Bucal, Ministério da Saúde. Projeto SB Brasil 2003 - Condições de saúde bucal da população brasileira: resultados principais. Brasília: Ministério da Saúde; 2004

5. Acs G, Pretzer S, Foley M, Ng MW. Perceived outcomes and parental satisfaction following dental rehabilitation under general anesthesia. Pediatr Dent 2001; 23:419-23.
6. Acs G, Shulman R, Ng MW, Chussid S. The effect of dental rehabilitation on the body weight of children with early childhood caries. Pediatr Dent 1999; 21:109-13.

7. Ayhan H, Suskan E, Yildirim S. The effect of nursing or rampant caries on height, body weight and head circumference. J Clin Pediatr Dent 1996; 20:209-12

8. Low W, Tan S, Schwartz S. The effect of severe caries on the quality of life in young children. Pediatr Dent 1999; 21:325-6.

9. Filstrup SL, Briskie D, Fonseca M, Lawrence L, Wandera A, Inglehart MR. Early childhood caries and quality of life: child and parent perspectives. Pediatr Dent 2003; 25:431-40.

10. Feitosa S, Colares V, Pinkham J. The psychosocial effects of severe caries in 4-year-old children in Recife, Pernambuco, Brazil. Cad Saúde Pública 2005; 21:1550-6. 
11. Anderson HK, Drummond BK, Thomson WM. Changes in aspects of children's oral-health-related quality of life following dental treatment under general anaesthesia. Int J Paediatr Dent 2004; 14:317-25.

12. Agostini FG, Flaitz CM, Hicks MJ. Dental emergencies in a university-based pediatric dentistry postgraduate outpatient clinic: a retrospective study. ASDC J Dent Child 2001; 68:316-21.

13. Traebert J, Almeida ICS, Garghetti C, Marcenes W. Prevalência, necessidade de tratamento e fatores predisponentes do traumatismo na dentição permanente de escolares de 11 a 13 anos de idade. Cad Saúde Pública 2004; 20:403-10.

14. Marques LS, Barbosa CC, Ramos-Jorge ML, Pordeus IA, Paiva SM. Prevalência da maloclusão e necessidade de tratamento ortodôntico em escolares de 10 a 14 anos de idade em Belo Horizonte, Minas Gerais, Brasil: enfoque psicossocial. Cad Saúde Pública 2005; 21:1099-106.

15. Cerqueira MN, Teixeira SC, Naressi SCM, Ferreira APP. Ocorrência de fissuras labiopalatais na cidade de São José dos Campos-SP. Rev Bras Epidemiol 2005; 8:161-6.

16. Oliveira BH, Milbourne P. Fluorose dentária em incisivos superiores permanentes em crianças de escola pública do Rio de Janeiro, RJ. Rev Saúde Pública 2001; 35:276-82.

17. Cortes MI, Marcenes W, Sheiham A. Impact of traumatic injuries to the permanent teeth on the oral health-related quality of life in 12-14-year-old children. Community Dent Oral Epidemiol 2002; 30:193-8.

18. Oliveira CM, Sheiham A. Orthodontic treatment and its impact on oral health-related quality of life in Brazilian adolescents. J Orthod 2004; 31:20-7.

19. Marques LS, Ramos-Jorge ML, Paiva SM, Pordeus IA. Malocclusion: esthetic impact and quality of life among Brazilian schoolchildren. Am J Orthod Dentofacial Orthop 2006; 129:424-7.

20. Peres KG, Latorre MRDO, Peres MA, Traebert J, Panizzi M. Impacto da cárie e da fluorose dentária na satisfação com a aparência e com a mastigação de crianças de 12 anos de idade. Cad Saúde Pública 2003; 19:323-30.

21. Nikias M. Oral disease and quality of life. Am J Public Health 1985; 75:11-2.

22. Jokovic A, Locker D, Stephens M, Kenny D, Tompson B, Guyatt G. Validity and reliability of a questionnaire for measuring child oral-health-related quality of life. J Dent Res 2002; 81:459-63.

23. Locker D, Jokovic A, Stephens M, Kenny D, Tompson B, Guyatt G. Family impact of child oral and oro-facial conditions. Community Dent Oral Epidemiol 2002; 30:438-48.

24. World Health Organization. WHOQOL - measuring quality of life. The World Health Organization quality of life instruments. Geneva: World Health Organization; 1997.

25. McGrath C, Broder H, Wilson-Genderson M. Assessing the impact of oral health on the life quality of children: implications for research and practice. Community Dent Oral Epidemiol 2004; 32:81-5.

26. Seidl EM, Zannon CMLC. Qualidade de vida e saúde: aspectos conceituais e metodológicos. Cad Saúde Pública 2004; 20:580-8.
27. Assumpção Jr. FB, Kuczynski E, Sprovieri MH, Aranha EMG. Escala de avaliação de qualidade de vida: (AUQEI - Autoquestionnaire Qualité de Vie Enfant Imagé) validade e confiabilidade de uma escala para qualidade de vida em crianças de 4 a 12 anos. Arq Neuropsiquiatr 2000; 58:119-27.

28. Wallander JL, Schmitt M, Koot HM. Quality of life measurement in children and adolescents: issues, instruments, and applications. J Clin Psychol 2001; 57:571-85.

29. Sevenhuysen GP, Trumble-Waddell J. A new perspective on quality of life. J Clin Epidemiol 1997; 50:231-2.

30. Pal DK. Quality of life assessment in children: a review of conceptual and methodological issues in multidimensional health status measures. J Epidemiol Community Health 1996; 50:391-6.

31. Fleck MPA, Leal OF, Louzada S. Desenvolvimento da versão em português do instrumento de avaliação de qualidade de vida da OMS (WHOQOL-100). Rev Bras Psiquiatr 1999; 21:19-28.

32. Ware Jr. JE, Sherbourne CD. The MOS 36-item short-form health survey (SF-36). I. Conceptual framework and item selection. Med Care 1992; 30:473-83.

33. Ciconelli RM, Ferraz MB, Santos W, Meinão I, Quaresma MR. Tradução para a língua portuguesa e validação do questionário genérico de avaliação de qualidade de vida SF-36 (Brasil SF-36). Rev Bras Reumatol 1999; 39:143-50.

34. Bergner M, Bobbitt RA, Carter WB, Gilson BS. The Sickness Impact Profile: development and final revision of a health status measure. Med Care 1981; 19:787-805.

35. Ruperto N, Ravelli A, Pistorio A, Malattia C, Cavuto S, Gado-West L, et al. Cross-cultural adaptation and psychometric evaluation of the Childhood Health Assessment Questionnaire (CHAQ) and the Child Health Questionnaire (CHQ) in 32 countries. Review of the general methodology. Clin Exp Rheumatol 2001; 19(4 Suppl 23):S1-9.

36. Manificat S, Dazord A, Cochat P, Nicolas J. Evaluation of the quality of life in pediatrics: how to collect the point of view of children. Arch Pediatr 1997; 4:1238-46.

37. Landgraf JM, Maunsell E, Speechley KN, Bullinger M, Campbell S, Abetz L, et al. Canadian-French, German and UK versions of the Child Health Questionnaire: methodology and preliminary item scaling results. Qual Life Res 1998; 7:433-45.

38. Machado CS, Ruperto N, Silva CH, Ferriani VP, Roscoe I, Campos LM, et al. The Brazilian version of the Childhood Health Assessment Questionnaire (CHAQ) and the Child Health Questionnaire (CHQ). Clin Exp Rheumatol 2001; 19(4 Suppl 23): S25-9.

39. Cohen LK, Jago JD. Toward the formulation of sociodental indicators. Int J Health Serv 1976; 6:681-98.

40. Reisine ST. Theoretical considerations in formulating sociodental indicators. Soc Sci Med [A] 1981; 15:745-50.

41. Weintraub JA. Uses of oral health related quality of life measures in public health. Community Dent Health 1998; 15:8-12. 
42. Locker D. Applications of self-reported assessments of oral health outcomes. J Dent Educ 1996; 60:494-500.

43. Hayes C. The use of patient based outcome measures in clinical decision making. Community Dent Health 1998; 15:19-21.

44. Atchison KA, Dolan TA. Development of the Geriatric Oral Health Assessment Index. J Dent Educ 1990; 54:680-7.

45. Leao A, Sheiham A. The development of a sociodental measure of dental impacts on daily living. Community Dent Health 1996; 13:22-6.

46. Slade GD, Spencer AJ. Development and evaluation of the Oral Health Impact Profile. Community Dent Health 1994; 11:3-11.

47. Slade GD. Derivation and validation of a shortform Oral Health Impact Profile. Community Dent Oral Epidemiol 1997; 25:284-90.

48. Slade GD, editor. Oral impacts on daily performances: measuring oral health and quality of life. North Carolina: Department of Dental Ecology, School of Dentistry, University of North Carolina; 1997.

49. Broder HL, Slade G, Caine R, Reisine S. Perceived impact of oral health conditions among minority adolescents. J Public Health Dent 2000; 60:189-92.

50. Oliveira CM, Sheiham A. The relationship between normative orthodontic treatment need and oral health-related quality of life. Community Dent Oral Epidemiol 2003; 31:426-36.

51. Ferreira CA, Loureiro CA, Araújo VE. Propriedades psicométricas de indicador subjetivo aplicado em crianças. Rev Saúde Pública 2004; 38:445-52.

52. Kuczynski E, Assumpção Jr. FB. Definições atuais sobre o conceito de qualidade de vida na infância e adolescência. Pediatr Mod 1999; 35:73-8.

53. Broder HL, Janal M, Wilson-Genderson M, Reisine ST, Phillips C. Reliability and validity of the Child Oral Health Impact Profile. J Dent Res 2005; 83(Special Issue A):2652.

54. Gherunpong S, Tsakos G, Sheiham A. Developing and evaluating an oral health-related quality of life index for children; the CHILD-OIDP. Community Dent Health 2004; 21:161-9.

55. Talekar BS, Rozier RG, Slade GD. Development of an OHRQoL instrument for preschool children. J Dent Res 2004; 83(Special Issue A):686.

56. Locker D. Measuring oral health: a conceptual framework. Community Dent Health 1988; 5:3-18.

57. Jokovic A, Locker D, Stephens M, Kenny D, Tompson B, Guyatt G. Measuring parental perceptions of child oral health-related quality of life. J Public Health Dent 2003; 63:67-72.

58. Jokovic A, Locker D, Tompson B, Guyatt G. Questionnaire for measuring oral health-related quality of life in eight- to ten-year-old children. Pediatr Dent 2004; 26:512-8.

59. Jokovic A, Locker D, Guyatt G. Short forms of the Child Perceptions Questionnaire for 11-14-yearold children (CPQ11-14): development and initial evaluation. Health Qual Life Outcomes 2006; 4:4.

60. Talekar BS, Rozier RG, Zeldini LP. Spanish version of the Early Childhood Oral Health Impact Scale. J Dent Res 2005; 83(Special Issue A):2653.
61. Tesch F, Leão A, Oliveira BH. Brazilian version of the Early Childhood Oral Health Impact Scale. J Dent Res 2006; 85(Special Issue A):1984.

62. Li S, Loignon J, Veronneau J, Allison P. Cross-cultural validation of a French infant oral health impact questionnaire. J Dent Res 2006; 85(Special Issue B):1200.

63. Adulyanon S, Vourapukjaru J, Sheiham A. Oral impacts affecting daily performance in a low dental disease Thai population. Community Dent Oral Epidemiol 1996; 24:385-9.

64. Tubert-Jeannin S, Pegon-Machat E, GremeauRichard C, Lecuyer MM, Tsakos G. Validation of a French version of the CHILD-OIDP index. Eur J Oral Sci 2005; 113:355-62.

65. French DJ, Carroll A, Christie MJ. Health-related quality of life in Australian children with asthma: lessons for the cross-cultural use of quality of life instruments. Qual Life Res 1998; 7:409-19.

66. Eiser C. Children's quality of life measures. Arch Dis Child 1997; 77:350-4.

67. Riley AW. Evidence that school-age children can self-report on their health. Ambul Pediatr 2004; 4(4 Suppl):371-6.

68. Buller H. Assessment of quality of life in the younger child: the use of an animated computer program. J Pediatr Gastroenterol Nutr 1999; 28: 53-5.

69. Eiser C, Morse R. Can parents rate their child's health-related quality of life? Results of a systematic review. Qual Life Res 2001; 10:347-57.

70. Drotar D. Validating measures of pediatric health status, functional status, and health-related quality of life: key methodological challenges and strategies. Ambul Pediatr 2004; 4(4 Suppl):358-64.

71. Jokovic A, Locker D, Stephens M, Guyatt G. Agreement between mothers and children aged 11-14 years in rating child oral health-related quality of life. Community Dent Oral Epidemiol 2003; 31: 335-43.

72. Jokovic A, Locker D, Guyatt G. How well do parents know their children? Implications for proxy reporting of child health-related quality of life. Qual Life Res 2004; 13:1297-307.

73. Theunissen NC, Vogels TG, Koopman HM, Verrips GH, Zwinderman KA, Verloove-Vanhorick SP, et al. The proxy problem: child report versus parent report in health-related quality of life research. Qual Life Res 1998; 7:387-97.

74. Stein RE, Jessop DJ. The impact on family scale revisited: further psychometric data. J Dev Behav Pediatr 2003; 24:9-16.

75. Juniper EF, Guyatt GH, Feeny DH, Ferrie PJ, Griffith LE, Townsend M. Measuring quality of life in the parents of children with asthma. Qual Life Res 1996; 5:27-34.

76. Gherunpong S, Tsakos G, Sheiham A. The prevalence and severity of oral impacts on daily performances in Thai primary school children. Health Qual Life Outcomes 2004; 2:57.

Recebido em 17/Jul/2006

Versão final reapresentada em 15/Mai/2007

Aprovado em 23/Mai/2007 